\title{
Bioactive Compounds in Legumes and Their Germinated Products
}

\author{
PEI-YIN Lin AND HSI-MeI LAI* \\ Department of Agricultural Chemistry, National Taiwan University, No. 1, Roosevelt Road, \\ Sec. 4, Taipei 10617, Taiwan
}

\begin{abstract}
Nineteen domestic legume varieties, including 6 soybeans, 7 black soybeans, 4 azuki beans, and 2 mung beans, were evaluated for contents of dietary fiber, total phenolics, and flavonoids. Nine varieties of legumes (black soybean TN6, TN3, BM, and WY; soybean KS1, KS2, and KS8; azuki bean AKS5 and AKS6) were good sources of bioactive compounds and were selected for germination tests. After short- and long-term germinations, the bioactive compounds were determined and compared with compositions of isoflavones in soybeans. The reducing power of legumes correlated well with their total flavonoid contents $\left(r^{2}=0.9414\right)$, whereas less correlation was found between reducing power and total phenolics contents $\left(r^{2}=0.6885\right)$. The dark-coat seeds, such as azuki beans and black soybeans, contained high amounts of phenolic compounds and contributed to high antioxidative ability, whereas their phenolics content and antioxidative abilities significantly decreased after shortterm germination due to losses of pigments in the seed coats. After long-term germination, the contents of bioactive compounds (total phenolics and flavonoids) increased again and the ratio of aglycones to total isoflavones significantly increased in black soybeans. TN3 and TN6 seeds and their longterm germinated seeds and AKS5 seeds were identified as the legume samples that might have the highest antioxidant ability according to the results of chemometric analysis. Selection of the right legume varieties combined with a suitable germination process could provide good sources of bioactive compounds from legumes and their germinated products for neutraceutical applications.
\end{abstract}

KEYWORDS: Legume; germination; phenolics; flavonoids; isoflavones; antioxidative activity

\section{INTRODUCTION}

Legumes are present in almost every diet throughout the world because they are good sources of starch, dietary fiber, protein, lipids, and minerals. In addition to their nutritive value, legumes contain significant quantities of phenolic and polyphenolic compounds such as phenolic acids, flavonoids, and lignins $(1,2)$. Phenolic compounds present in legumes include anthocyanidin pigments such as delphinidin, cyanidin, pelargonidin, malvidin, and petunidin (3). These have antioxidative effects that are beneficial to human health $(4,5)$. The legume attracting much attention recently is the soybean, which contains a high concentration of the isoflavones, one class of phytoestrogens (6). Twelve isoflavones from soybean have been separated and identified, which are categorized by their functional groups into four major subgroups: aglycones (daidzein, genistein, glycitein), glucosides (daidzin, genistin, glycitin), malonyl glucosides, (malonyl daidzin, malonyl genistin, malonyl glycitin), and acetyl glucosides (acetyl daidzin, acetyl genistin, acetyl glycitin) (7, 8 ). Recent studies indicate that isoflavones, especially the aglycones, have benefits against cardiovascular diseases and cancers and function by acting as antiestrogens $(9,10)$.

* Corresponding author (telephone +886-2-33664816; fax +886-223633123; e-mail hmlai@ntu.edu.tw).
In addition to the seeds, germinated bean sprouts are also served as staple vegetables and used in soups, salads, and side dishes in many Asian countries. In the natural environment, seed sprouts survive during germination by enhancing their defensive responses through phenolics biosynthesis (11). Germination may cause changes in the nutrients, including functional substances, through aerobic respiration and biochemical metabolism. Sprouting also removes antinutrients such as enzyme inhibitors in the seeds, thus making sprouts safe for the diet (12). The contents and compositions of isoflavones vary greatly among soybean sprouts, immature soybeans, and mature soybeans. The ratio of aglycones to total isoflavones decreased from the sprouts, to the immature beans, to the mature beans, whereas the total isoflavones and ratio of glucosides to total isoflavones decreased in the reverse order according to Nakamura et al. (13). Zhu et al. (14) investigated the effect of germination on the isoflavone content in two soybean varieties and reported that the maximum content of isoflavones can be controlled by the degree of germination of soybean seeds.

Recently, legumes are gaining interest because they are excellent sources of bioactive compounds and can be important sources of ingredients for uses in functional foods and other applications. It is well-known that the content of bioactive compounds of legumes is generally affected by planting 
environmental and genetic factors such as cultivar, cultivation year, cultivation location, and temperature $(8,15)$. The contents and compositions of phenolic compounds and isoflavones also vary with storage time and germination period of seeds (11). Hoeck et al. (15) reported that the differences between soybean cultivars with the highest and lowest total and individual isoflavone contents were relatively consistent among the different environmental growing conditions. Therefore, looking for the good cultivars, combined with optimal germination periods of legumes to obtain high content of bioactive compounds, is critical if legumes are to become a competitive source of phytonutrients.

The objectives of this study were first to compare the contents of dietary fiber and antioxidative compounds and their antioxidative abilities in 19 varieties of domestic legumes, including 6 soybeans, 7 black soybeans, 4 azuki beans, and 2 mung beans. The legumes containing high amounts of dietary fiber and phytochemicals were further germinated, and their antioxidative compounds and abilities were reevaluated. In addition, the contents and compositions of isoflavones at different germination stages of soybeans were analyzed. The information provided in this study is valuable for the ingredient selection of legumes for the applications of functional foods.

\section{MATERIALS AND METHODS}

Legumes. Nineteen domestic breeding legumes, 6 soybean [Glycine $\max (\mathrm{L})$.$] cultivars (KS1, KS2, KS3, KS5, KS6, and KS8), 7$ black soybean cultivars (TN3, TN5, TN6, KS7, BM, WY, and DP), 4 azuki bean (Vigna angularis) cultivars (AKS5, AKS6, AKS7, and AKS8), and 2 mung bean (Vigna radiate) cultivars (GTN5 and VC), were kindly provided from Kaohsiung and Tainan District Agricultural Research and Extension Stations in Taiwan and used as samples in this study. The seeds were ground by a Knifetec 1095 sample mill (Foss Tecator, Sweden), sieved through a 40 mesh screen, and then stored at $4{ }^{\circ} \mathrm{C}$ until use.

Proximate Composition Analysis. The content of moisture, ash, crude fat, and crude protein of samples was determined according to AACC methods 44-31A, 08-16, 30-25, and 46-12, respectively (16). The content of dietary fiber was determined by using the enzymatic gravity method AACC 32-07 operated on a Fibertec System E (Foss Tecator, Sweden). Soluble, insoluble, and total dietary fiber of samples was determined. All of the analyses were carried out in triplicate and expressed as the mean.

Seed Germination. Seeds were soaked in water at $35^{\circ} \mathrm{C}$ for $5 \mathrm{~h}$, drained, and then layered on a plastic seed germination box on which a layer of cheesecloth had been laid. The box was covered with a sheet of aluminum foil to keep it in complete darkness, and seeds were germinated in an incubator at $25^{\circ} \mathrm{C}$. Two germination stages, shortterm germination (1 day) with 3-6 mm length of sprouts and longterm germination (4 days) with $3-6 \mathrm{~cm}$ length of sprouts, were cultivated. The sprouts were harvested and immediately freeze-dried. The dried sprouts were ground into fine powder with a Knifetec 1095 sample mill and stored at $4{ }^{\circ} \mathrm{C}$ until use.

Phenolics and Flavonoids Determination. The ground bean or sprout powder $(5 \mathrm{~g})$ was suspended in $80 \%$ methanol solution (100 $\mathrm{mL}$ ) and extracted at $60{ }^{\circ} \mathrm{C}$ in a water bath with continuous shaking for $2 \mathrm{~h}$. The extracted solvent was filtered through Whatman no. 541, and the filtrate was used for the assays of total phenolics, total flavonoids, and reducing power. The extraction recovery of legume samples was determined by repeating extraction from the residues left from pre-extraction, and the total phenolics of extracts were determined. The extraction recovery is $95.45 \pm 0.35 \%$ when extracted only one time.

Total phenolics of the sample was measured as gallic acid equivalents (micrograms of gallic acid per milligram of dry meal) according to the method of Taga et al. (17). The filtrate $(100 \mu \mathrm{L})$ was diluted with 100 $\mu \mathrm{L}$ of $\mathrm{MeOH} / 0.3 \% \mathrm{HCl}(6: 4, \mathrm{v} / \mathrm{v})$, mixed with $2 \% \mathrm{Na}_{2} \mathrm{CO}_{3}$ solution (2 $\mathrm{mL}$ ), and kept for $2 \mathrm{~min}$. After the addition of $100 \mu \mathrm{L}$ of $50 \%$ Folin-
Ciocalteu's reagent (Sigma, St. Louis, MO) to the mixture, the filtrate was incubated for $30 \mathrm{~min}$ at room temperature in the dark. Then the filtrate was vortexed, and the absorbance at $750 \mathrm{~nm}$ was measured using a UA-160A UV spectrophotometer (Shimadzu, Japan). Total flavonoids of the sample was expressed as catechin equivalents (micrograms of of catechin per milligram of dry meal) and measured by using a colorimetric assay developed by Zhishen et al. (18). Appropriate diluted filtrate $(1 \mathrm{~mL})$ was transferred into a $10 \mathrm{~mL}$ volumetric flask containing $4 \mathrm{~mL}$ of distilled water. The following solutions were added into the volumetric flask at the specific time: $0.3 \mathrm{~mL}$ of $5 \% \mathrm{NaNO}_{2}$ solution at time $0,0.3 \mathrm{~mL}$ of $10 \% \mathrm{AlCl}_{3}$ at $5 \mathrm{~min}$, and $2 \mathrm{~mL}$ of $1 \mathrm{~N} \mathrm{NaOH}$ at $6 \mathrm{~min}$. The flask contents were then immediately diluted with $2.4 \mathrm{~mL}$ of distilled water and mixed. The absorbance at $510 \mathrm{~nm}$ of mixture was measured using the UV spectrophotometer.

Antioxidative Ability Assay. The antioxidative ability of samples was evaluated by the assays of the reducing power, which was expressed as ascorbic acid equivalents (micrograms of ascorbic acid per milligram of dry meal) (19) and free radical scavenging capacity, which used 2,2-diphenyl-1-picrylhydrazyl (DPPH) as free radical substrate (20). For the assay of reducing power, $1 \mathrm{~mL}$ of filtrate was mixed with 2.5 $\mathrm{mL}$ of phosphate buffer $(\mathrm{pH} 6.6)$ and $2.5 \mathrm{~mL}$ of $1 \%$ of $\mathrm{K}_{3} \mathrm{Fe}(\mathrm{CN})_{6}$ solution, followed by incubation at $50{ }^{\circ} \mathrm{C}$ for $20 \mathrm{~min}$. The reaction was then stopped by adding $10 \%$ trichloroacetic acid $(2.5 \mathrm{~mL})$, followed by centrifuging at $3000 \mathrm{rpm}$ for $10 \mathrm{~min}$. The supernatant $(2.5 \mathrm{~mL})$ was mixed with distilled water $(2.5 \mathrm{~mL})$ and $1 \% \mathrm{FeCl}_{3}$ solution $(0.5 \mathrm{~mL})$, and the absorbance at $700 \mathrm{~nm}$ was measured. For the DPPH scavenging capacity assay, the appropriate concentration of extracted sample was added into DPPH methanol solution, which contained a final concentration of $0.1 \mathrm{mM}$ DPPH. After incubation at room temperature for 30 $\mathrm{min}$, the absorbance at $519 \mathrm{~nm}$ of the reacting mixture was measured. The scavenging capability of DPPH was calculated by the following equation:

$$
\% \text { DPPH scavenging }=\left[\left(\mathrm{OD}_{\text {control }}-\mathrm{OD}_{\text {sample }}\right) / \mathrm{OD}_{\text {control }}\right] \times 100 \%
$$

The absorbance at $519 \mathrm{~nm}$ of DPPH methanol solution without added samples was measured and used as the absorbance of control sample. The parameter $\mathrm{EC}_{50}$, which reflects the concentration of sample extracts that had 50\% DPPH scavenging capability, was also determined.

Isoflavones Extraction and Determination. The extraction method for isoflavones from soybeans and their sprouts was modified from that of Kao et al. (21). Two grams of ground seed or sprout powder was extracted with $19 \mathrm{~mL}$ of solvent of acetonitrile/distilled water (10: $9, \mathrm{v} / \mathrm{v}$ ) at room temperature for $2 \mathrm{~h}$. After filtration through Whatman no. 541 , the filtrate was evaporated under $40{ }^{\circ} \mathrm{C}$ almost to dryness. The dried material was redissolved in $10 \mathrm{~mL}$ of $80 \%$ methanol and stored at $-20{ }^{\circ} \mathrm{C}$ until use. The determination of isoflavones was performed by HPLC using a Hitachi L-6000 system with a Hitachi L-7455 photodiode array detector (PDA) (Hitachi Ltd., Japan). A 250 $\mathrm{mm} \times 4.6 \mathrm{~mm}$ i.d., $5 \mu \mathrm{m}$, YMC-pack ODS-AM-303 column (YMC Co., Ltd., Japan) was used to carry out the separation. A linear HPLC gradient was employed: solvent A was $0.01 \%$ TFA in acetonitrile, and solvent B was $0.01 \%$ TFA in distilled water. Solvent A was increased from 10 to $40 \%$ over $60 \mathrm{~min}$, increased from 40 to $90 \%$ in $3 \mathrm{~min}$, and then decreased from 90 to $10 \%$ in $2 \mathrm{~min}$. The injection volume of sample was $20 \mu \mathrm{L}$ with a flow rate of $1.0 \mathrm{~mL} / \mathrm{min}$ during the measurement. The PDA detector monitored the absorbance of solution at $260 \mathrm{~nm}$. UV spectra were recorded, and area responses were integrated by using D-7000 HPLC System Manager software (Hitachi, Ltd., Japan). Both aglycones and their conjugated isoflavones, including daidzein, daidzin, acetyl daidzin, genistein, genistin, acetyl genistin, malonyl genistin, malonyl glycitin (LC Laboratories Co., Woburn, MA), glycitein, glycitin, acetyl glycitin, and malonyl daidzin (Nagara Science Co., Ltd., Gifu, Japan), were used as standards. The extraction recovery of aglycones and conjugated isoflavones had been carried out by spiking 12 standards into a soybean sample, and then the extraction procedures were proceeded. The isoflavones of extracts were quantified by HPLC, and the average recoveries of isoflavones were $96.75 \pm 0.88 \%$.

Statistical Analysis. Every assay in this study was run in triplicate, and the results are presented as mean \pm standard deviation. The effects of legume type, legume variety, and stage of seed germination on the 
Table 1. Proximate Compositions of 19 Legumes $^{a}$

\begin{tabular}{|c|c|c|c|c|c|c|c|c|}
\hline \multirow[b]{3}{*}{ variety } & \multirow{3}{*}{$\begin{array}{l}\text { moisture } \\
(\mathrm{g} / 100 \mathrm{~g})\end{array}$} & \multicolumn{7}{|c|}{$\mathrm{g} / 100 \mathrm{~g}$, dry meal } \\
\hline & & \multirow[b]{2}{*}{ ash } & \multirow[b]{2}{*}{ fat } & \multirow[b]{2}{*}{ protein } & \multicolumn{3}{|c|}{ dietary fiber } & \multirow[b]{2}{*}{ residue } \\
\hline & & & & & $\overline{\mathrm{SDF}}$ & IDF & $\overline{T D F}$ & \\
\hline \multicolumn{9}{|l|}{ soybean } \\
\hline KS1 & $9.81 \pm 0.10$ & $5.41 \pm 0.01 \mathrm{de}$ & $15.09 \pm 0.27 b c$ & $36.48 \pm 1.31 d$ & 3.31 & 26.01 & 29.32 & 13.70 \\
\hline KS2 & $9.24 \pm 0.06$ & $5.50 \pm 0.00 \mathrm{~cd}$ & $11.86 \pm 0.03 f$ & $43.90 \pm 0.67 a$ & 1.75 & 24.57 & 26.32 & 14.42 \\
\hline KS3 & $9.43 \pm 0.03$ & $5.83 \pm 0.02 a$ & $13.70 \pm 0.10 \mathrm{de}$ & $43.78 \pm 0.11 \mathrm{a}$ & 1.53 & 21.09 & 22.62 & 14.07 \\
\hline KS5 & $8.74 \pm 0.07$ & $5.77 \pm 0.04 b$ & $14.76 \pm 0.10 c$ & $41.24 \pm 0.23 b$ & 1.89 & 22.42 & 24.31 & 13.92 \\
\hline KS6 & $8.87 \pm 0.02$ & $5.75 \pm 0.04 b$ & $15.53 \pm 0.06 \mathrm{~b}$ & $38.26 \pm 1.00 \mathrm{c}$ & 2.28 & 23.33 & 25.61 & 14.85 \\
\hline KS8 & $8.61 \pm 0.12$ & $5.78 \pm 0.03 a b$ & $15.26 \pm 0.30 \mathrm{bc}$ & $44.57 \pm 0.32 a$ & 3.01 & 19.53 & 22.54 & 11.85 \\
\hline \multicolumn{9}{|c|}{ black soybean } \\
\hline TN3 & $10.51 \pm 0.09$ & $5.26 \pm 0.01 f$ & $13.48 \pm 0.18 \mathrm{e}$ & $40.88 \pm 0.37 b$ & 3.99 & 26.32 & 30.31 & 10.07 \\
\hline TN5 & $10.93 \pm 0.46$ & $5.28 \pm 0.02 f$ & $14.17 \pm 0.06 \mathrm{~d}$ & $43.89 \pm 0.57 a$ & 2.54 & 24.36 & 26.90 & 9.76 \\
\hline TN6 & $10.16 \pm 0.06$ & $5.72 \pm 0.02 b$ & $9.36 \pm 0.06 \mathrm{~g}$ & $42.62 \pm 0.04 a$ & 4.63 & 24.64 & 29.27 & 13.03 \\
\hline KS7 & $9.39 \pm 0.21$ & $5.54 \pm 0.02 \mathrm{c}$ & $17.81 \pm 0.69 \mathrm{a}$ & $38.79 \pm 1.38 \mathrm{c}$ & 1.14 & 21.65 & 22.79 & 15.07 \\
\hline BM & $8.04 \pm 0.18$ & $5.51 \pm 0.03 \mathrm{~cd}$ & $15.09 \pm 0.19 b c$ & $41.53 \pm 0.07 b$ & 3.08 & 22.65 & 25.73 & 12.14 \\
\hline WY & $7.96 \pm 0.11$ & $5.48 \pm 0.01 \mathrm{cde}$ & $13.82 \pm 0.58 \mathrm{de}$ & $41.10 \pm 0.04 b$ & 3.45 & 21.75 & 25.20 & 14.40 \\
\hline $\mathrm{DP}$ & $7.94 \pm 0.10$ & $5.42 \pm 0.03 e$ & $18.01 \pm 0.13 a$ & $42.00 \pm 0.16 b$ & 2.41 & 19.36 & 21.77 & 12.80 \\
\hline \multicolumn{9}{|l|}{ azuki bean } \\
\hline AKS5 & $11.97 \pm 0.03$ & $3.81 \pm 0.11 \mathrm{i}$ & $0.62 \pm 0.02 i$ & $22.83 \pm 0.24 f$ & 0.97 & 19.31 & 20.28 & 52.46 \\
\hline AKS6 & $12.35 \pm 0.03$ & $3.77 \pm 0.06 \mathrm{i}$ & $0.73 \pm 0.07 i$ & $22.02 \pm 2.73 f g$ & 2.67 & 17.95 & 20.62 & 52.86 \\
\hline AKS7 & $12.16 \pm 0.02$ & $3.70 \pm 0.03 i$ & $0.87 \pm 0.07 i$ & $22.98 \pm 0.28 f$ & 1.09 & 20.70 & 21.79 & 50.66 \\
\hline AKS8 & $12.21 \pm 0.10$ & $3.62 \pm 0.02 k$ & $0.85 \pm 0.04 i$ & $19.19 \pm 0.29 \mathrm{~h}$ & 1.30 & 26.32 & 27.62 & 48.72 \\
\hline \multicolumn{9}{|l|}{ mung bean } \\
\hline GTN5 & $11.36 \pm 0.01$ & $3.88 \pm 0.04 h$ & $0.97 \mathrm{~h} \pm 0.21 \mathrm{i}$ & $26.75 \pm 0.39 \mathrm{e}$ & 0.86 & 13.07 & 13.93 & 54.47 \\
\hline VC & $12.26 \pm 0.04$ & $3.96 \pm 0.02 \mathrm{~g}$ & $1.45 \pm 0.03 \mathrm{~h}$ & $21.18 \pm 0.67 \mathrm{~g}$ & 0.64 & 13.93 & 14.57 & 58.84 \\
\hline
\end{tabular}

${ }^{a}$ Mean values in each column sharing the same letter are not significantly different $(p<0.05) \cdot{ }^{b}$ Residue $(\%)=100-($ ash + fat + protein + TDF $) \%$.

content of antioxidative compounds and antioxidative ability of legumes were analyzed by the analysis of variance (ANOVA) and Duncan's new multiple-range test $(p<0.05)$ by using SAS software developed by the SAS Institute, Inc. (Cary, NC). The chemometic analysis methods of principal component analysis (PCA) and cluster analysis were applied to summarize all variables to a mathematical model, which describes a linear combination of original variables and is responsible for the antioxidative ability of legume samples. Both PCA and cluster analysis with $k$ means were performed using public domain $\mathrm{R}$ software v. 2.2.1 (22).

\section{RESULTS AND DISCUSSION}

Proximate Composition of Legumes. The proximate compositions of 19 legumes are shown in Table 1. Soybeans and black soybeans contained mainly protein with a range of $36.48-$ $44.57 \%$ and dietary fiber with a range of $21.77-30.31 \%$. Next were fat with a range of $9.36-18.01 \%$ and nutritive carbohydrates (mainly starch, shown as residue in Table 1) with a range of $9.76-15.07 \%$, and least is ash $(\approx 5 \%)$ in the dry matter. The proximate composition of azuki and mung beans contained mainly nutritive carbohydrate and dietary fiber, which ranged from 48.72 to $58.84 \%$ and from 13.93 to $27.62 \%$, respectively. Azuki and mung beans contained much less fat than soybeans, and their ash contents were slightly less than that of soybeans. The content of total dietary fiber (TDF) and the ratio of soluble dietary fiber (SDF) to the TDF depended on legume type and variety. In general, black soybeans contained high amounts of TDF and SDF, whereas two mung bean varieties contained the least amount of TDF among the 19 legume varieties studied in this study. Soybeans KS1 and KS8, black soybeans TN3, TN6, $\mathrm{BM}$, and WY, and azuki AKS6 were good sources of TDF and SDF. According to the report of the National Cholesterol Education Program (NCEP) Expert Panel on Detection, Evaluation, and Treatment, increased soluble fiber intake (10-25 $\mathrm{g}$ /day) is included in the therapeutic life changes in low-density lipoprotein (LDL)-lowering therapy and recommended for persons who have a relatively high risk of coronary heart disease $(23,24)$.
Total Phenolics and Flavonoids of Legumes. Most azuki beans and some of the black soybeans (TN3 and TN6) contained high amounts of total phenolics and flavonoids. In azuki beans, the ratios of total flavonoids to total phenolics were all $>30 \%$. It has been reported that azuki bean seed coats mainly contained proanthocyanins, which are a group of polyphenolic bioflavonoids with remarkable radical scavenging activities in vitro $(25,26)$. Compared to the soybeans, black soybeans TN3 and TN6 contained very high amounts of total phenolics and flavonoids and concentrated amounts of anthocyanins in dark seed coats (27). The legumes with stronger reducing power had higher amounts of total flavonoids with good coefficient of determination $\left(r^{2}=0.9414\right)$, whereas the correlations between total phenolics and total flavonoids and between total phenolics and reducing power were less and their coefficients of determination were 0.6364 and 0.6885 , respectively. However, removing the data of TN3 and TN6 from the data pool, the coefficients of determination were significantly improved to 0.8245 and 0.8626 , respectively. These results revealed that the phenolic compounds of legumes were unevenly distributed in the seeds, especially for the legumes with dark seed coats. However, the reducing power of legumes was dependent on the content of phenolic compounds; specifically, it was closely dependent on the content of total flavonoids.

Antioxidative Ability of Legumes after Germination. On the basis of the contents of soluble dietary fiber and antioxidative properties determined previously, soybeans KS1, KS2, and KS8, black soybeans TN3, TN6, BM, and WY, and azuki beans AKS5 and AKS6 were selected and germinated. The germination process changed total phenolics and flavonoids contents of selected legumes, and their reducing powers were also altered (Figure 1). It was found that the total phenolics and total flavonoids of legumes which had dark seed coats (AKS5, AKS 6 , TN3, and TN6) and high amounts of phenolic compounds in mature seeds significantly decreased after short-term germination. However, their antioxidative compounds increased after long-term germination. The changes of total phenolics and 


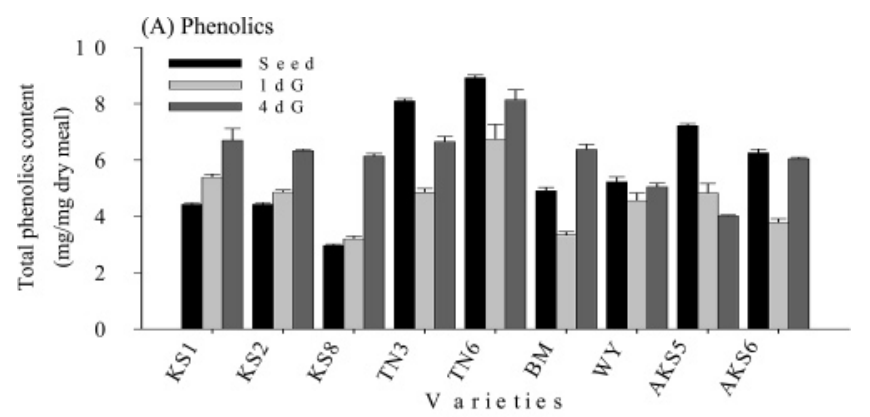

Table 2. DPPH Scavenging Ability of Mature and Germinated Seeds of Selected Legumesa

\begin{tabular}{cccc}
\hline & \multicolumn{3}{c}{$\mathrm{EC}_{50}{ }^{\mathrm{b}}(\mathrm{mg} / \mathrm{mL})$} \\
\cline { 2 - 4 } variety & seed & 1-day germinated & 4-day germinated \\
\hline soybean & & & \\
KS1 & $7.7 \pm 0.7 \mathrm{~g}$ & $7.5 \pm 0.4 \mathrm{e}$ & $14.9 \pm 0.0 \mathrm{a}$ \\
KS2 & $6.5 \pm 0.1 \mathrm{f}$ & $10.7 \pm 0.7 \mathrm{~b}$ & $4.8 \pm 0.2 \mathrm{gh}$ \\
KS8 & $7.8 \pm 0.2 \mathrm{~cd}$ & $7.9 \pm 0.4 \mathrm{~d}$ & $4.7 \pm 0.2 \mathrm{~h}$ \\
black soybean & & & \\
TN3 & $0.5 \pm 0.0 \mathrm{mn}$ & $2.5 \pm 0.0 \mathrm{j}$ & $0.9 \pm 0.01 \mathrm{~m}$ \\
TN6 & $1.0 \pm 0.01$ & $\mathrm{NA} \mathrm{C}^{\mathrm{c}}$ & $2.7 \pm 0.1 \mathrm{j}$ \\
BM & $2.4 \pm 0.1 \mathrm{jk}$ & $5.3 \pm 0.1 \mathrm{f}$ & $3.6 \pm 0.1 \mathrm{c}$ \\
WY & $0.8 \pm 0.01 \mathrm{~m}$ & $3.1 \pm 0.1 \mathrm{i}$ & $2.1 \pm 0.0 \mathrm{k}$ \\
azuki bean & & & \\
AKS5 & $0.4 \pm 0.0 \mathrm{n}$ & $0.6 \pm 0.01 \mathrm{mn}$ & $2.5 \pm 0.0 \mathrm{jk}$
\end{tabular}

${ }^{a}$ Mean values sharing the same letter are not significantly different $(p<0.05)$. ${ }^{b} \mathrm{EC}_{50}$, the concentration $(\mathrm{mg} / \mathrm{mL})$ of sample extracts is needed to scavenge $50 \%$ of DPPH. ${ }^{c} \mathrm{NA}$, not available.

Table 3. Loadings for Four Principal Components of Legume Samples from Three Stages of Eight Selected Legume Varieties

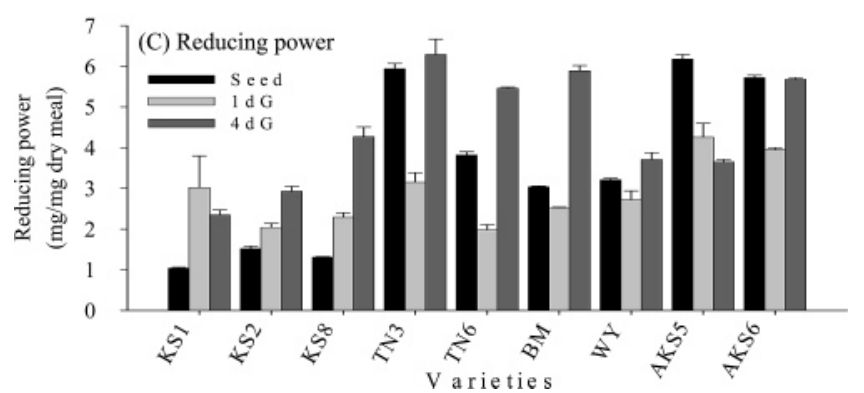

\begin{tabular}{lccrr}
\hline \multicolumn{1}{c}{ variable } & PC1 & PC2 & \multicolumn{1}{c}{ PC3 } & \multicolumn{1}{c}{ PC4 } \\
\hline phenolics (PHE) & -0.469 & -0.680 & -0.299 & 0.478 \\
flavonoids (FLA) & -0.519 & -0.016 & 0.855 & 0.003 \\
reducing power (RP) & -0.551 & -0.012 & -0.332 & -0.765 \\
EC $_{50}$ of DPPH scavenging (DPPH) & 0.455 & -0.733 & 0.264 & -0.431 \\
\hline
\end{tabular}

of AKS5 mature seed dry meal, which was 50- and 133-fold compared to pure tocopherol $(0.201 \mathrm{~g})$ and ascorbic acid $(0.076$ g), respectively (28).

PCA was applied on the data matrix of phenolics, flavonoids,

Figure 1. Antioxidative properties of mature seeds and germinated legumes: (A) total phenolics content; (B) total flavonoids content; (C) reducing power. Abbreviations: 1dG, 1-day germinated; 4dG, 4-day germinated.

flavonoids contents of germinated legumes also reflected the results of their reducing power. The decreases of the amounts of antioxidative compounds and antioxidative ability of shortterm-germinated legume products were attributed to the losses of pigmentation compounds in seed coats when the seeds were soaked and germinated. This could be seen by the colored cheesecloth layered on the bed of germination box. In contrast to the results of legumes with dark seed coats, the contents of total phenolics and flavonoids of soybeans were increased after germination and increased with increasing germination time. The same transitions were also investigated on the basis of the results of reducing power of legumes and their germinated products. These findings were attributed to the biochemical metabolism of seeds during germination, which might produce some secondary plant metabolites such as anthocyanins and flavonoids or release aglycones from conjugated glycosides from seed coats and cotyledons due to the enzymatic activation (11).

The 2,2-diphenyl-1-picrylhydrazyl (DPPH) method had good repeatability and has been widely used to determine antiradical/ antioxidative activity of purified phenolic compounds as well as natural plant extracts. Table 2 shows the $\mathrm{EC}_{50}$ of legumes (milligrams per milliliter) required to scavenge $50 \%$ of DPPH at the assay conditions. It was found that seeds from azuki bean AKS5 and black soybeans TN3, WY, and TN6 had good antiradical/antioxidant activities, as well as the germinated seeds such as 1-day-germinated $(1 \mathrm{dG})$ AKS5 and 4-day-germinated (4dG) TN3, which had compatible antiradical/antioxidative activities. To scavenge $1 \mathrm{~g}$ of DPPH radical required $10.14 \mathrm{~g}$ reducing power, and $\mathrm{EC}_{50}$ of $\mathrm{DPPH}$ scavenging of legume samples from three stages (matured seed, short- and long-term germination) of eight selected legume varieties (KS1, KS2, KS8, TN3, TN6, BM, WY, and AKS5). The data matrix was standardized by an autoscaling procedure to give the same importance to all variables. The measurements of TN6 $1 \mathrm{dG}$ were not included because the $\mathrm{EC}_{50}$ of DPPH was not available in this study. PCA identified two uncorrelated linear combinations of measurements that account for $86.4 \%$ of the total variation in the original four measurements. The first principal component (PC1) and the second principal component (PC2) account for 69.5 and $16.9 \%$ of the total variation, respectively. From the loading vectors (Table 3), PC1 was expressed by the following equation:

$\mathrm{PC} 1=-0.469($ phenolics $)-0.519($ flavonoids $)-0.551$
$($ reducing power $)+0.455\left(\mathrm{EC}_{50}\right.$ of $\left.\mathrm{DPPH}\right)$

PC1 might account for the antioxidant ability of legume samples. The smaller PC1 indicates the legume samples with the stronger antioxidative ability. Cluster analysis has been performed to identify legume samples exhibiting similar structural characteristics of PC scores. The score plot of the first two principal components reveals that there are three distinct groups of legume samples (Figure 2). TN3 seed, TN3 4dG, TN6 seed, TN6 4dG, and AKS5 seed were identified as the group that might have the highest antioxidant ability.

Isoflavones of Soybeans and Effects of Germination. One of the most exciting developments in the field of nutraceuticals and functional foods is the beneficial biological activity of isoflavones from soybeans. The bioavailability and bioactivity of isoflavones are affected by their chemical structures. It appears that aglycones of daidzein and genistein are more 


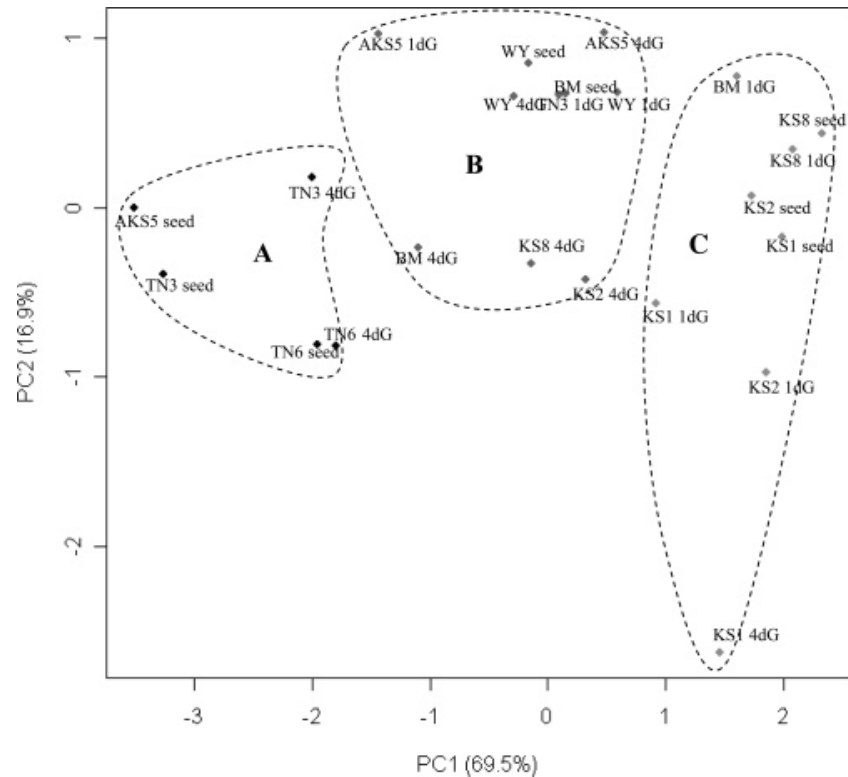

Figure 2. Score plot of the first two principal components (PC1 and PC2) for the legume samples from three stages of eight selected legume varieties.
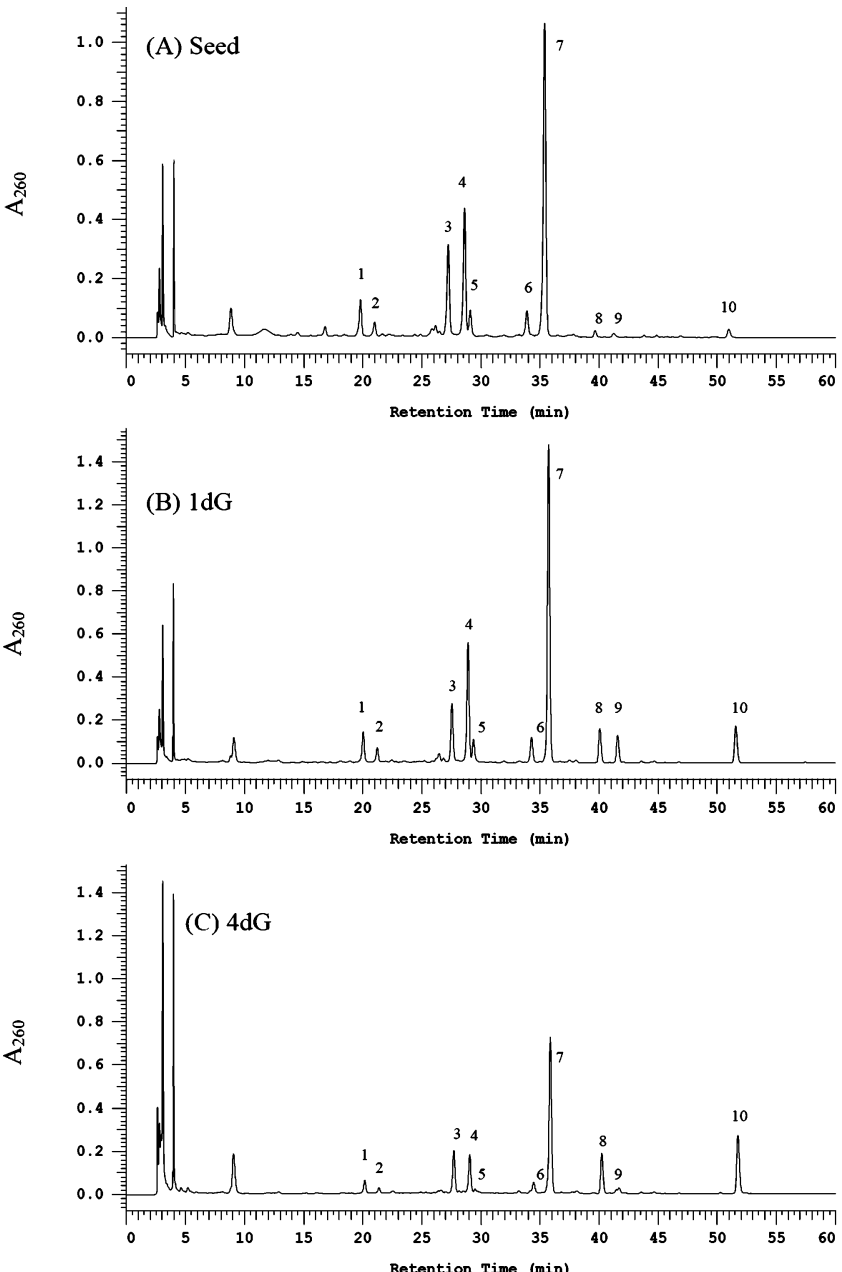

Figure 3. HPLC-PDA chromatograms of isoflavones in mature seeds and germinated black soybean TN3: (A) mature seeds; (B) 1-day germinated (1dG); (C) 4-day germinated (4dG). Peaks: 1, daizin; 2, glycitin; 3, genistin; 4, malonyl daidzin; 5 , malonyl glycitin; 6 , acetyl glycitin; 7, malonyl genistin; 8, daidzein; 9, glycitein; 10, genistein.

bioavailable than their conjugated forms in humans (29). The bioavailability also changed between isoflavone families, as daidzein was found to be more bioavailable than genistein in
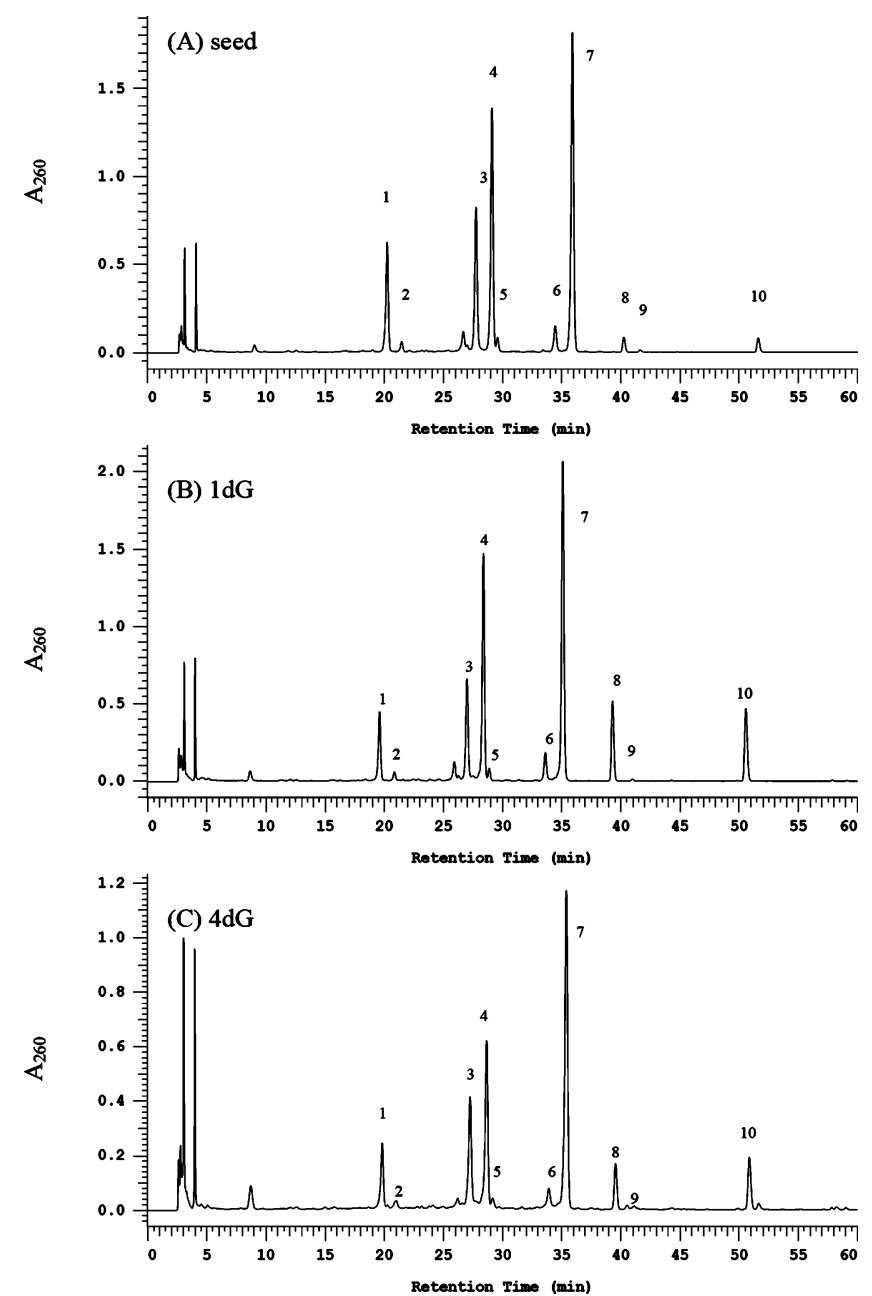

Figure 4. HPLC-PDA chromatograms of isoflavones in mature seeds and germinated soybean KS1: (A) mature seeds; (B) 1-day germinated (1dG); (C) 4-day germinated (4dG). Peaks: 1, daizin; 2, glycitin; 3, genistin; 4, malonyl daidzin; 5 , malonyl glycitin; 6 , acetyl glycitin; 7, malonyl genistin; 8, daidzein; 9, glycitein; 10, genistein.

adult women (30) and conjugated daidzein was more bioavailable than genistein conjugate in rats (31). These observations indicated that deglycosylation of soy isoflavones may significantly affect their bioavailabilities for humans. The chromatograms of isoflavones in black soybeans and soybeans are shown in Figures 3 and 4. Although the total contents of soybean isoflavones were dependent on the varieties and the stages of germination (Table 4), their isoflavones compositions were similar among the varieties. The main components of isoflavones were malonyl glucosides (from 56 to $76 \%$ of total isoflavones) in mature soybean seeds. The total isoflavones of whole seed extracts ranged from 1795 to $4333.51 \mu \mathrm{g} / \mathrm{g}$ of dry meal, being lowest for black soybean TN6 and highest for soybean KS1. Wang and Murphy (7) measured eight American and three Japanese soybean varieties and reported that the total isoflavone contents ranged from 2053 to $4216 \mu \mathrm{g} / \mathrm{g}$ and the major constituents were malonyl genistin, malonyl daidzin, and daidzin. In general, soybeans contained higher total isoflavones than black soybeans, except black soybean TN3, whereas aglycones were hardly found in mature black soybeans but small amounts of daidzein and genistein could be determined in mature soybeans KS1 and KS2.

The compositions of isoflavones were significantly changed after germination and influenced by the varieties and stages of germination. The aglycones, daidzein, genistein, and glycitein, 

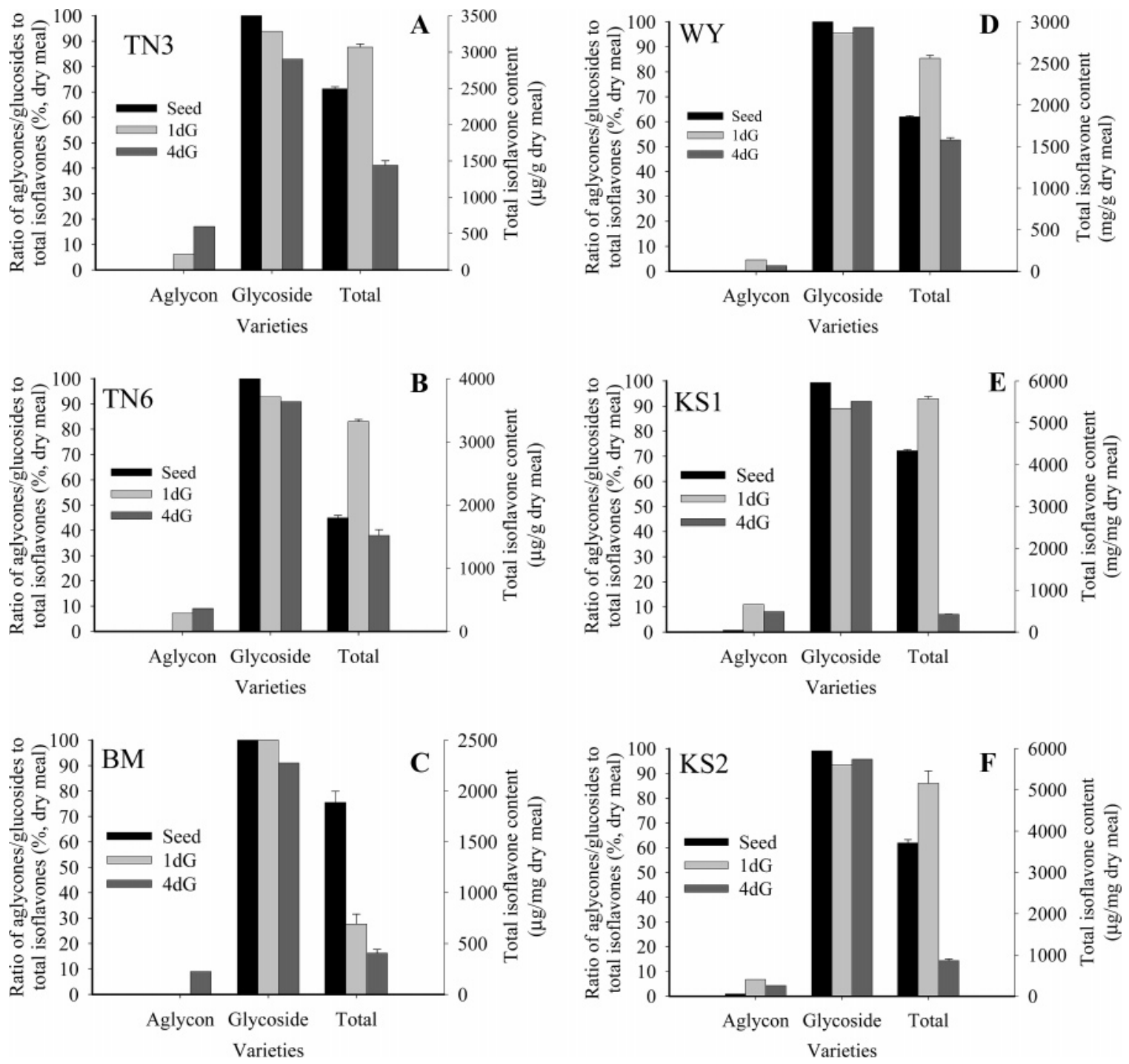

Figure 5. Composition changes of isoflavones of soybeans and black soybeans: (A) black soybean TN3; (B) black soybean TN6; (C) black soybean BM; (D) black soybean WY; (E) soybean KS1; (F) soybean KS2. Abbreviations: 1dG, 1-day germinated; 4dG, 4-day germinated.

significantly increased from 0.7 to $10 \%$ (KS1) and from 0.1 to $17 \%$ (TN6) after 1 day of germination. Total isoflavones also significantly increased in all soybeans after 1 day of germination. At the beginning of germination, not only aglycones releasing from glucosides through the catalysis of activated glucosidase in seeds but also isoflavones produced through the biosynthesis in the malonate and phenylpropanoid pathways (32) contributed to the boost in the amounts of total isoflavones. However, the amounts of total isoflavones and aglycones significantly decreased after 4 days of germination in all soybeans (Figure 5). Although the total isoflavones of black soybeans increased after short-term germination and then decreased after long-term germination, the aglycones of their sprouts increased with increasing germination time (Figure 5A-C). Zhu et al. (14) reported that the maximum amounts of total isoflavone, genistein, and daidzein and their $\beta$-glycoside conjugates were obtained when hypocotyl lengths of germinated seeds were 0.5 and $2.5 \mathrm{~mm}$ of var. Hutcheson $(2.491,1.500$, and $0.905 \mathrm{mg} / \mathrm{g}$ ) and var. Caviness $(2.78,1.523$, and $0.905 \mathrm{mg} / \mathrm{g})$, respectively.

The contents and compositions of bioactive compounds varied greatly between types of legumes and their varieties, due to genetic characteristics. Also, the compositions of isoflavones differed in each growth stage of soybeans (such as immature soybean and mature soybean) and the degree of seed germination (such as short- and long-term germination). Black soybeans (TN3 and TN6) and azuki beans (AKS5 and AKS6) had strong reducing power and high amounts of total flavonoids in mature seeds, partially attributed to the anthocyanins in dark seed coats. The total phenolics, total flavonoids, and reducing power of legumes with dark seed coats decreased significantly after shortterm germination, but they increased after long-term germination. The total isoflavone contents and the ratios of aglycones to total isoflavones of black soybeans (TN3, TN6, and BM) significantly increased after short-term germination. Soybeans (KS1 and KS2) contained high amounts of total isoflavones and aglycones in mature seeds, but their total isoflavone contents and the ratios of aglycones to total isoflavones significantly decreased after long-term germination compared to the shortterm germinated soybeans. The antioxidative components and antioxidative abilities of soybeans KS1 and KS2 increased with increasing germination time. Consuming the legumes and related products is becoming popular due to human health concerns. The findings in this study provide information for the selection of suitable legume types and varieties and proper germination stages to obtain the greatest potential functional ingredients from legume sources. 


\section{ACKNOWLEDGMENT}

We thank Dr. Yun-Ming Pong and Dr. Jeng-Min Chiou for useful discussion of principal component analysis and cluster analysis.

\section{LITERATURE CITED}

(1) Dabrowski, K. J.; Sosulski, F. W. Composition of free and hydrolyzable phenolic acids in the flours and hulls of 10 legume species. J. Agric. Food Chem. 1984, 32, 131-133.

(2) Dabrowski, K. J.; Sosulski, F. W. Composition of free and hydrolyzable phenolic acids in defatted flours of 10 oilseeds. $J$. Agric. Food Chem. 1984, 32, 128-130.

(3) Mazza, G.; Miniati, E. Anthocyanins in Fruits, Vegetables, and Grains; CRC Press: Boca Raton, FL, 1993; pp 2-10.

(4) Hill, C. Sources of natural antioxidants: oilseeds, nuts, cereals, legumes, animal products and microbial sources. In Antioxidant in Food: Practical Applications; Pokorny, J., Yanishlieva, N., Gordon, M., Eds.; CRC Press: Boca Raton, FL, 2001.

(5) Shahidi, F.; Naczk, M. Antioxidant properties of food phenolics. In Phenolics in Food and Neutraceuticals; CRC Press: Boca Raton, FL, 2004.

(6) Mazur, W. M.; Duke, J. A.; Wahala, K.; Rasku, S.; Adlercreutz, H. Isoflavonoids and lignans in legumes: Nutritional and health aspects in humans. J. Nutr. Biochem. 1998, 6, 193-200.

(7) Wang, H. J.; Murphy, P. A. Isoflavone composition of American and Japanese soybeans in Iowa: effects of variety, crop year, and location. J. Agric. Food Chem. 1994, 42, 1674-1677.

(8) Wang, H. J.; Murphy, P. A. Isoflavone content in commercial soybean foods. J. Agric. Food Chem. 1994, 42, 1666-1673.

(9) Albertazzi, P.; Purdie, D. W. The nature and utility of the phytoestrogens: a review of the evidence. Maturitas 2002, 42, 173-185.

(10) Ewies, A. A. A. Phytoestrogens in the management of the menopause: up-to-date. Obstet. Gynecol. Surv. 2002, 57, 306313.

(11) Randhir, R.; Lin, Y. T.; Shetty, K. Stimulation of phenolics, antioxidant and antimicrobial activities in dark germinated mung bean sprouts in response to peptide and phytochemical elicitors. Process Biochem. 2004, 39, 637-647.

(12) Mwikya, S. M.; Camp, J. V.; Rodriguez, R.; Huyghebaert, A. Effects of sprouting on nutrient and antinutrient composition of kidney beans (Phaseolus vulgaris var. Rose coco). Eur. Food Res. Technol. 2001, 212, 188-191.

(13) Nakamura, Y.; Kaihara, A.; Yoshii, K.; Tsumura, Y.; Ishimitsu, S.; Tonogai, Y. Content and composition of isoflavonoids in mature or immature beans and bean sprouts consumed in Japan. J. Health Sci. 2001, 47, 394-406.

(14) Zhu, D.; Hettiarachchy, N. S.; Horax, R.; Chen, P. Isoflavone contents in germinated soybean seeds. Plant Foods Hum. Nutr. 2005, 60, 147-151.

(15) Hoeck, J. A.; Fehr, W. R.; Murphy, P. A.; Welke, G. A. Influence of genotype and environment on isoflavone contents of soybean. Crop Sci. 2000, 40, 48-51.

(16) AACC. Approved Methods of the American Association of Cereal Chemists; AACC: St. Paul, MN, 1995.

(17) Taga, M. S.; Miller, E. E.; Pratt, D. E. Chia seeds as a source of natural antioxidants. J. Am. Oil Chem. Soc. 1984, 61, 928-931.

(18) Zhishen, J.; Mengcheng, T.; Jianming, W. The determination of flavonoid contents in mulberry and their scavenging effects on superoxide radicals. Food Chem. 1999, 64, 555-559.
(19) Yen, G. C.; Chen, H. Y. Antioxidant activity of various tea extracts in relation to their antimutagenicity. J. Agric. Food Chem. 1995, 43, 27-32.

(20) Kiitts, D. D.; Wijewickreme, A. N.; Hu, C. Antioxidant properties of North American ginseng extract. Mol. Cell. Biol. 2000, 203, $1-10$.

(21) Kao, F. J.; Su, N. W.; Lee, M. H. Effect of water-to-bean ratio on the contents and compositions of isoflavones in tofu. J. Agric. Food Chem. 2004, 52, 2277-2281.

(22) R Development Core Team. R: A Language and Environment for Statistical Computing; R Foundation for Statistical Computing: Vienna, Austria, 2005; ISBN 3-900051-07-0, URL http:// www.R-project.org.

(23) Cleeman, J. I. Executive summary of the Third Report of the National Cholesterol Education Program (NCEP) Expert Panel on detection, evaluation and treatment of high blood cholesterol in adults (Adult Treatment Panel III). JAMA-J. Am. Med. Assoc. 2001, 285, 2486-2497.

(24) Grundy, S. M.; Cleeman, J. I.; Merz, C. N.; Brewer, H. B., Jr.; Clark, L. T.; Hunninghake, D. B.; Pasternak, R. C.; Smith, S. C., Jr.; Stone, N. J. Coordinnating Committee of the National Cholesterol Education Program; National Heart, Lung and Blood Institute; American College of Cardiology Foundation; American Heart Association. Implications of recent clinical trials for the National Cholesterol Education Program Adult Treatment Panel III guidelines. J. Am. Coll. Cardiol. 2004, 44, 720-732.

(25) Ariga, T.; Koshiyama, I.; Fukushima, D. Antioxidative properties of procyanidins B-1 and B-3 from azuki beans in aqueous system. Agric. Biol. Chem. 1988, 52, 2717-2722.

(26) Ariga, T.; Hamano, M. Radical scavenging action and its mode in procyanidins B-1 and B-3 from azuki beans to peroxyl radicals. Agric. Biol. Chem. 1990, 54, 2499-2504.

(27) Choung, M. G.; Baek, I. Y.; Kang, S. T.; Han, W. Y.; Shin, D. C.; Moon, H. P.; Kang, K. H. Isolation and determination of anthocyanins in seed coats of black soybean (Glycine $\max (\mathrm{L}$.) merr.). J. Agric. Food Chem. 2001, 49, 5848-5851.

(28) Concepción, S.-M.; José, A. L.; Fulgencio, S.-C. A procedure to measure the antiradical efficiency of polyphenols. J. Sci. Food Agric. 1998, 76, 270-276.

(29) Hutchins, A. M.; Slavin, J. L.; Lampe, W. Urinary isoflavonoid phytoestrogen and lignan excretion after consumption of fermented and unfermented soy products. J. Am. Diet. Assoc. 1995, 95, 545-551.

(30) Xu, X.; Wang, H. J.; Murphy, P. A.; Hendrich, S. Daidzein is a more bioavailable soymilk isoflavone than is genistein in adult women. J. Nutr. 1994, 124, 825-832.

(31) King, R. A. Daidzein conjugates are more bioavailable than genistein conjugates in rats. Am. J. Clin. Nutr. 1998, 68, 1496S1499S.

(32) Hahlbrock, K.; Scheel, D. Physiology and molecular biology of phenylpropanoid metabolism. Plant Mol. Biol. 1989, 40, 347369.

Received for review January 2, 2006. Revised manuscript received March 25, 2006. Accepted March 30, 2006. This work was supported by Grants 93AS-5.1.3-FD-Z1 and 94AS-12.1.3-FD-Z1 from the Council of Agriculture Executive Yuan, Taiwan.

JF060002O 